\title{
INVESTIGATION OF NUCLEAR STRUCTURE BY QUASI-FREE SCATTERING WITH POLARIZED PROTONS ${ }^{\text {is }}$
}

\author{
F. FERNANDEZ ${ }^{1}$, Th.A.J. MARIS, C. SCHNEIDER and C.A.Z. VASCONCELLOS \\ Instituto de Fisica, Universidade Federal do Rio Grande do Sul, 90000 Porto Alegre, RS, Brazil
}

Received 4 March 1981

\begin{abstract}
Quasi-free scattering with polarized protons can be a sensitive method to probe certain spin correlations in the nuclear wave function. This is demonstrated by the comparison of the effective polarizations following from the shell model and a deuteron cluster model for the nuclei ${ }^{14} \mathrm{~N}$ and ${ }^{6} \mathrm{Li}$.
\end{abstract}

In medium-energy quasi-free scattering processes [1], the nuclear target nucleon is in general polarized before it is knocked out. One can measure this polarization $[2,3]$ in $(p, 2 p)$ reactions using a polarized proton beam, because the matrix element for mediumenergy proton-proton scattering has a strong spin dependence.

During the last few years several $(p, 2 p)$ coincidence experiments with polarized protons have been performed $[4,5]$ and the measured asymmetries have clearly confirmed the occurrence of large polarizations of the nuclear nucleon. The most extensive measurements [5] have been made for the nuclei ${ }^{16} \mathrm{O}$ and ${ }^{40} \mathrm{Ca}$; for these nuclei and in geometries for which the on-shell impulse approximation and the factorization [1] are expected to be good, the measured effective polarizations are in reasonable agreement with the theoretical expectations [3,5] following from the singleparticle shell model and calculated in the distorted wave impulse approximation (DWIA).

In this note we would like to point out that the effective polarization is caused by correlations of the spins and orbits of the nuclear nucleons and that therefore its measurement may give direct information on the structure of the ground state of the target

\footnotetext{
is Work partially supported by Financiadora de Estudos e Projetos (FINEP) and Conselho Nacional de Desenvolvimento Científico e Tecnológico (CNPq).

${ }^{1}$ On leave of absence from the Institut für Theoretische Physik, Tübingen, West Germany.
}

nucleus and/or of the involved final state of the residual nucleus.

To demonstrate this, we compare for the nuclei ${ }^{14} \mathrm{~N}$ and ${ }^{6} \mathrm{Li}$ the shell model predictions with the ones following from a model in which the valence nucleons have deuteron cluster correlations [6]. The effective polarizations following from these two models differ in sign and size.

At present we shall consider only coplanar geometries; under these conditions the effective polarization is orthogonal to the scattering plane and is given by [2]

$P_{\text {eff }}(k)=\frac{\Sigma_{m_{\mathrm{f}} m_{\mathrm{i}}}\left(\left|g_{m_{\mathrm{f}} m_{\mathrm{i}}}^{\prime(+)}(k)\right|^{2}-\left|g_{m_{\mathrm{f}} m_{\mathrm{i}}^{\prime(-)}}(k)\right|^{2}\right)}{\Sigma_{m_{\mathrm{f}} m_{\mathrm{i}}}\left(\left|g_{m_{\mathrm{f}} m_{\mathrm{i}}}^{\prime(+)}(k)\right|^{2}+\left|g_{m_{\mathrm{f}} m_{\mathrm{i}}}^{\prime(-)}(k)\right|^{2}\right)}$.

$g_{m_{\mathrm{f}} m_{\mathrm{j}}}^{( \pm)}(k)$ is the distorted momentum amplitude which may be written [7]:

$$
\begin{aligned}
& g_{m_{\mathrm{f}} m_{\mathrm{i}}}^{( \pm)}(k)=(2 \pi)^{-3 / 2} \\
& \quad \times \int \mathrm{e}^{-\mathrm{i} k \cdot r} D(r)\left\langle j_{\mathrm{f}} m_{\mathrm{f}} ; A-1\left|a^{( \pm)}(r)\right| j_{\mathrm{i}} m_{\mathrm{i}} ; A\right\rangle \mathrm{d}^{3} r ;
\end{aligned}
$$

where $\left\langle j_{\mathrm{i}} m_{\mathrm{i}} ; A\right\rangle$ and $\left|j_{\mathrm{f}} m_{\mathrm{f}} ; A-1\right\rangle$ denote the initial and final nuclear wave functions characterized by total angular momenta $j_{\mathrm{i}}$ and $j_{\mathrm{f}}$ and projections $m_{\mathbf{i}}$ and $m_{\mathrm{f}}$; the $a^{( \pm)}(r)$ are the annihilation operators of a nucleon with position $r$ and spin projections $( \pm)$; all angular momenta are quantized orthogonal to the scattering plane. The distorting function $D(r)$ represents the effects of multiple scattering of the incident and emer- 
gent particles by the rest of the nucleus.

In the shell model calculations we have used harmonic oscillator wave functions with a width parameter determinated to reproduce the rms radius measured by electron scattering [8] on the target nucleus. The ground states of ${ }^{14} \mathrm{~N}$ and ${ }^{6} \mathrm{Li}$ are considered to have a valence neutron and proton in $j=1 / 2$ and $j=3 / 2$ states, which couple with their cores, ${ }^{13} \mathrm{C}\left(j_{\mathrm{f}}=1 / 2\right)$ and ${ }^{5} \mathrm{He}\left(j_{\mathrm{f}}=3 / 2\right)$, respectively, to give $j_{\mathrm{i}}=1$. The final states are ${ }^{13} \mathrm{C}\left(j_{\mathrm{f}}=1 / 2\right)$ and ${ }^{5} \mathrm{He}\left(j_{\mathrm{f}}=3 / 2\right)$, each having one neutron outside a closed subshell or shell.

In the cluster model calculations we assume that the target nucleus consists of the inert spectator cores ${ }^{12} \mathrm{C}$ and ${ }^{4} \mathrm{He}$ and two correlated valence particles forming a deuteron cluster. In this model the wave function for the initial state may be written as

$\psi_{\mathrm{i}}=\not A[\chi($ Core $) \phi(\mathrm{d}) F(R)]$,

where $A$ is the antisymmetrization operator. The function $\phi(d)$, which describes the internal behaviour of the deuteron cluster, is chosen to be

$\phi(\mathrm{d})=\exp \left(-\frac{1}{2} \alpha^{2} \sum_{i=1}^{2}\left(r_{i}-R\right)^{2}\right) \xi(\sigma, \tau)$

$R$ denoting the deuteron center-of-mass coordinate. The spherical symmetric relative motion function $F(R)$ we have taken to be proportional to $R^{2}$ $\times \exp \left(-\beta^{2} R^{2}\right)$, with the usual factor $R^{2}$ of the cluster model prescription for valence particles in the p-shell [9]. $\xi(\sigma, \tau)$ is the spin-one-isospin-zero wave function.

The core wave functions in expression (3), as well as the corresponding final ${ }^{13} \mathrm{C}$ and ${ }^{5} \mathrm{He}$ ground states, are assumed to be identical to the ones of our shell model calculation. The size parameter $\alpha$ of the deuteron was varied between $1.9 \mathrm{fm}$ (the free value) and $1.4 \mathrm{fm}$, as from fits to cluster calculations [10] there seems to be some evidence for a contraction of the deuteron in the nucleus. For each value of $\alpha$ the parameter $\beta$ of the deuteron center-of-mass wave function was computed to reproduce the same density as in the shell model. Because of the values of the parameters and the shape of the wave function $F(R)$, there is on the average only a small core-deuteron overlap, so that exchange effects between these can be neglected.

In the computations we have assumed that the total center-of-mass of the system coincides with the core center-of-mass and is not affected by the knock-

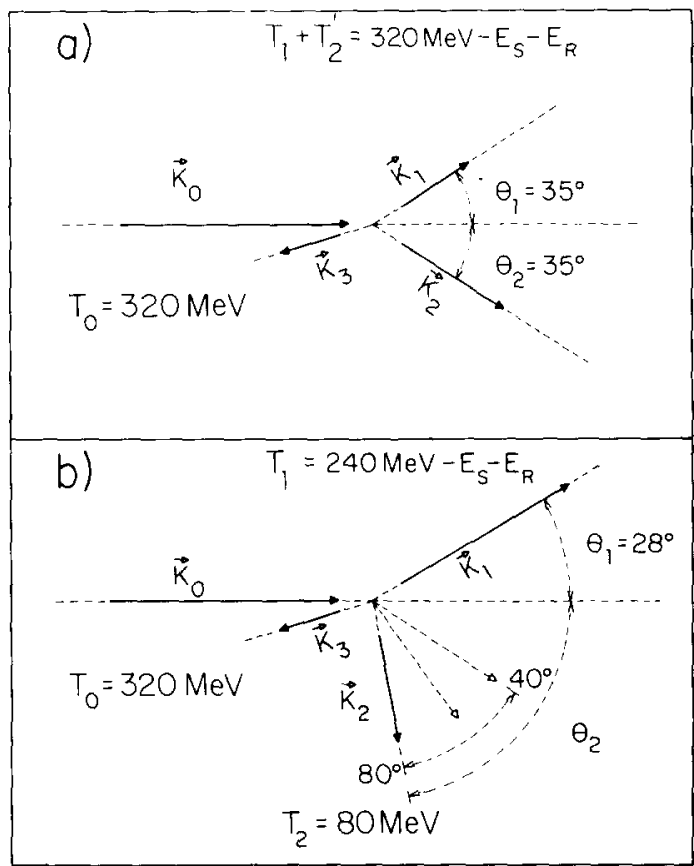

Fig. 1. The coplanar kinematics used in our calculations. The incoming and emerging protons are denoted by 0,1 and $2 . T$ means kinetic energy; $E_{\mathrm{S}}$ is the nuclear separation energy and $E_{\mathrm{R}}$ is the (small) recoil energy of the residual nucleus. In geometry (a) only the sharing of the available energy between the outgoing protons is varied. In geometry (b) the angle of proton 2 is essentially the only parameter varied.

out process (inert-core approximation). This assumption results in a strong simplification but causes an error of the order $A^{-1}$ in the squared overlap integrals; in particular in the case of ${ }^{6} \mathrm{Li}$ this may not seem to be a good approximation. However, for effective polarization calculations this error is considerably reduced as only ratios between momentum distributions enter. For our present purpose the approximation is sufficient, because the qualitative behaviour of the effective polarization is certainly not changed.

With the wave functions described above we have calculated the effective polarization for the ${ }^{14} \mathrm{~N}(\mathrm{p}, 2 \mathrm{p}){ }^{13} \mathrm{C}$ and ${ }^{6} \mathrm{Li}(\mathrm{p}, 2 \mathrm{p}){ }^{5} \mathrm{He}$ reactions for 320 $\mathrm{MeV}$ incoming protons in the two different coplanar kinematical situations shown in fig. 1.

The distorted wave calculations have been performed in the WKB approximation with a square-well spin-independent nuclear optical potential with real and imaginary parts calculated as in ref. [3], where 


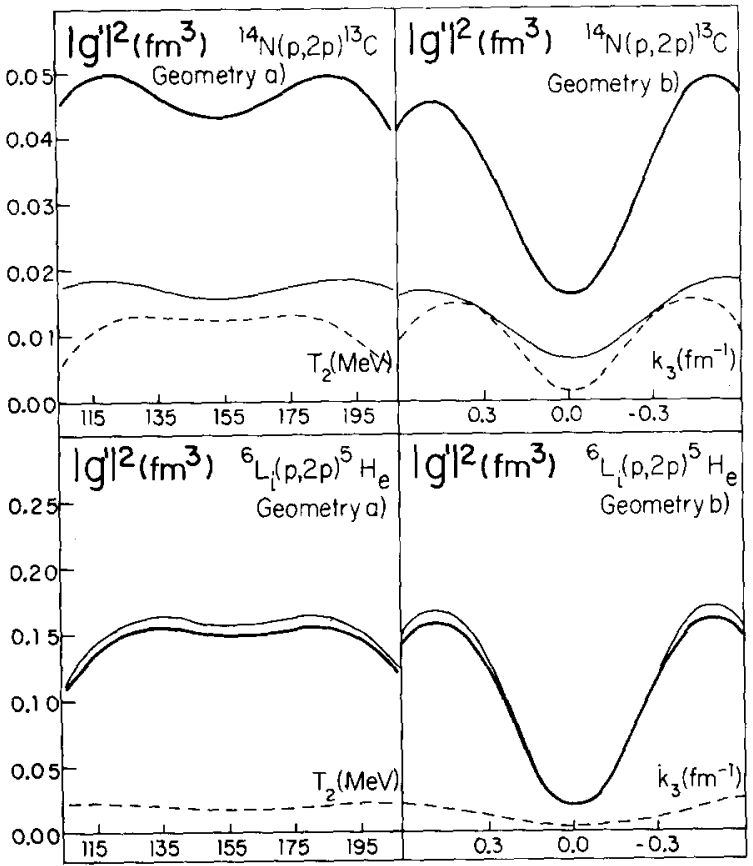

Fig. 2. The distorted momentum distributions. In each subfigure the thick line corresponds to the shell model; the full and dashed thin lines correspond to the cluster model with deuteron radii of $1.9 \mathrm{fm}$ and $1.5 \mathrm{fm}$, respectively.

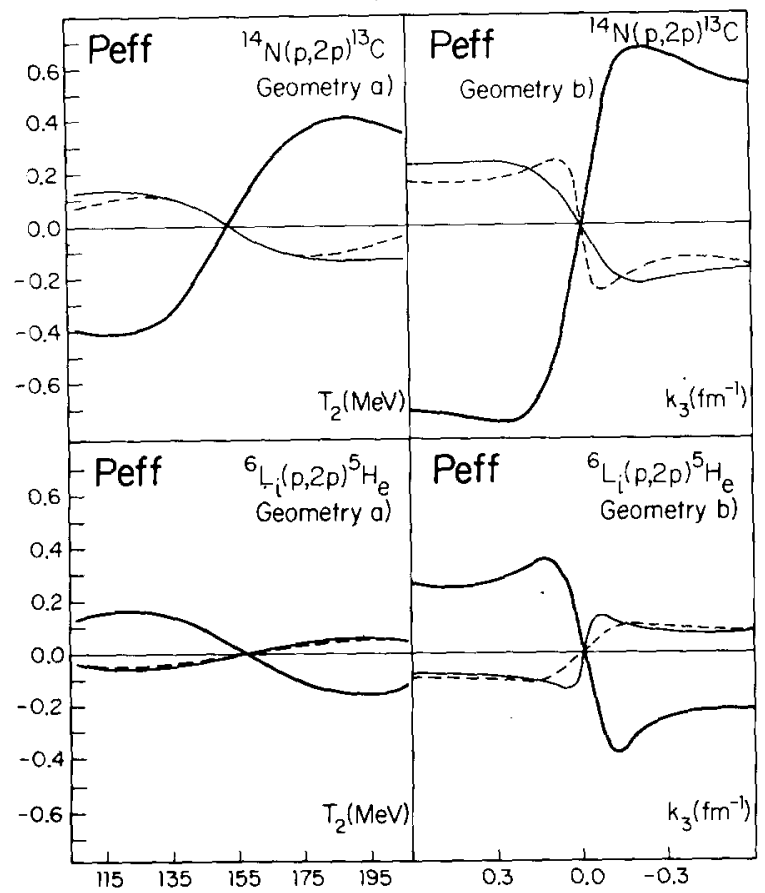

Fig. 3. The effective polarization; the meaning of the lines is the same as in fig. 2 . also typical values may be found; experience has shown $[3,5,11]$ that for the present energies the effective polarization is only slightly affected by such approximations.

Typical results are shown in figs. 2 and 3. One observes that the momentum distributions (fig. 2), which are the quantities measured in experiments with unpolarized protons, are not suitable to detect a cluster structure. The shapes of the distributions for the shell and cluster model are quite similar and their relative magnitudes depend sensitively on the choice of adjustable parameters. Anyhow, calculated absolute values of quasi-free cross sections are known to be very unreliable because of optical model and wave function uncertainties. On the other hand, fig. 3 shows that the effective polarizations for the two models differ in sign and size in all cases; they are only slightly influenced by reasonable parameter variations.

The present calculations are mainly meant as examples to show the effect of the nuclear structure on the effective polarizations, but nevertheless we would like to make some remarks on the specific results obtained:

(1) The differences between the effective polarizations for the pure shell model and the shell model plus cluster correlations are not difficult to understand. In the $j-j$ coupled shell model the effective polarization is caused by the correlations, in the initial nucleus, of the spin of the knocked-out proton with its momentum; as shown in ref. [3] this is a result of the spinorbit coupling and distortion effects in the selected asymmetric kinematics. In the cluster model there are no correlations of this type in the initial nucleus, because the internal and center-of-mass wave functions of the deuteron represent both $l=0$ states. However, because of the deuteron spin wave function with $S=1$, the spin of the knocked-out proton is parallel to the spin of the remaining valence neutron. This neutron has a momentum which has a tendency to be opposite to that of the knocked-out proton, because both momenta were anticorrelated in the deuteron. Using the distortion arguments of ref. [3] but now applied to the final nucleus, one finds again that the remaining neutron is effectively polarized but oppositely to the knocked-out proton in the shell model case, because of its opposite momentum. This polarization carries over to the knocked-out proton through the mentioned $S=1$ correlation, which explains the difference in sign of the effective polarization of the knockedout proton in the two models. 
The difference in size of the polarizations is caused by the fact that in the cluster model the momentum of the remaining valence neutron is not exactly opposite to the one of the knocked-out proton but is smeared by the center-of-mass motion of the deuteron.

(2) The polarizations in the ${ }^{6} \mathrm{Li}$ case are about $-1 / 2$ times the corresponding ones for ${ }^{14} \mathrm{~N}$, in agreement with the known trend [7] of the effective polarizations for $p_{3 / 2}$ and $p_{1 / 2}$ single-particle states. That this is also true for our cluster model follows from the preceding argument.

(3) The present method of investigation might give a clearer indication for a cluster structure than the use of reactions in which a cluster is supposed to be directly knocked out. In that case the quasi-free hypothesis is poor, because the cluster must be deformed in the nucleus. Furthermore, the possibilities of formation and break-up of the finally observed cluster before, during and after the knock-out process and its large size complicate the reaction mechanism, casting doubt on the use of the distorted wave approximation and on the interpretation of the results.

The given examples show that quasi-free scattering with polarized particles can be a sensitive method to measure certain spin correlations in the nuclear wave function. In general, to test a given wave function, one may calculate the expected effective polarization and compare this with experiment.

One of us (FF) wants to thank the members of the Instituto de Fisica of the UFRGS for the kind hospitality extended to him.

\section{References}

[1] T. Berggren and H. Tyrén, Ann. Rev. Nucl. Sci. 16 (1966) 153.

[2] Th.A.J. Maris, Nucl. Phys. 9 (1958/59) 577.

[3] G. Jacob, Th.A.J. Maris, C. Schneider and M.R. Teodoro, Phys. Lett. 45B (1973) 181; Nucl. Phys. A257 (1976) 517.

[4] V.S. Nadejdin, N.I. Petrov and V.I. Satarov, JINR, El7559 (Dubna, 1973); JINR, El-10820 (Dubna, 1977).

[5] P. Kitching et al., Phys. Rev. Lett. 37 (1976) 1600; P. Kitching et al., Nucl. Phys. A340 (1980) 423.

[6] K. Wildermuth and Y.C. Tang, A unified theory of the nucleus (Academic Press, New York, 1977).

[7] Th.A.J. Maris, M.R. Teodoro and C.A.Z. Vasconcellos, Nucl. Phys. A322 (1979) 461.

[8] R. Herman and R. Hof stadter, High-energy electron scattering tables (Stanford U.P., 1960).

[9] J.V. Noble, Phys. Rev. C9 (1974) 1209.

[10] J.Y. Grossiord et al., Phys. Rev. Lett. 32 (1974) 173.

[11] C. Schneider, Nucl. Phys. A300 (1978) 313. 